\title{
Doxycycline at Low Concentrations Could Influence Your Experimental Results When Working With Prostate Cancer Cell Lines
}

\author{
Maria Brattsand* \\ Department of Medical Biosciences, Pathology, Umeå University, Umeå, Sweden \\ *Corresponding author: Maria Brattsand, Department of Medical Biosciences, Pathology, Umeå University, Umeå, \\ Sweden
}

\section{ARTICLE INFO}

Received: 慧 June 05, 2020

Published: 䋖 June 17, 2020

Citation: Maria Brattsand. Doxycycline at Low Concentrations Could Influence Your Experimental Results When Working With Prostate Cancer Cell Lines. Biomed J Sci \& Tech Res 28(2)-2020. BJSTR. MS.ID.004636.

\section{ABSTRACT}

Doxycycline is a tetracycline derivate commonly used in various inducible expression systems to study the function of different proteins. In this study, we show that 22Rv1 and PC3, two different, commonly used prostate cancer cell lines are very sensitive to this antibiotic and addition of the drug at inductive concentrations can affect cells in ways that can be misinterpreted as effects from the protein studied. Therefore, proper controls are very important in order to avoid conclusions drawn by artifacts caused by experimental conditions.

\section{Short Communication}

A commonly used method to study the function of a certain gene product is to either overproduce or knock down the expression of the gene of interest in a cell line and then study the effects of this expression in different aspects [1]. The setup for these kinds of experiments is either to use a vector having a constantly active promoter, or an inducible expression system. There are several benefits of using inducible systems. If the gene of interest causes some kind of harm to the cell, you will automatically get a selection against the cells that are expressing increased levels of the harmful product. It could then be very difficult in getting enough material to study the exact effects of the gene you want to study. Using an inducible system in this case makes it possible to cultivate the cells to get enough material before inducing expression of the gene of interest. The same is true if you want to study a gene that is essential for growth of the cell and use an inducible knock down system. In many cases, you may need a cell line that expresses increased/decreased levels of a certain protein for a prolonged period. In stably transfected cell lines, the DNA from the transfected plasmid integrates into the genome of the cell. As integration of the genetic material could result in disruption of another gene, several different clones need to be analyzed to ensure that effects observed after transfection indeed results from changes in expression of the gene of interest. Using an inducible system, the parental noninduced cell is the perfect control of the induced one.

This is only valid, though, if the agent used to induce the expression is not by itself affecting the cells studied [2]. Low concentrations of tetracycline specifically inhibit mitochondrial protein synthesis, and prolonged exposure to the drug results in a proliferation arrest and an accumulation of cells in the G1 phase of the cell cycle [3]. In this work, we want to emphasize the need for proper controls using inducible expression systems to avoid drawing the wrong conclusions from the results obtained.

\section{Materials and Methods}

\section{Cell Lines}

22Rv1 (CRL-2505) and PC3 (CRL-1435) were purchased from American Type Culture Collection (ATCC), and maintained in complete RPMI 1640 with Glutamax I (Fisher Scientific), supplemented with $10 \%$ heat-inactivated Tet System approved fetal bovine serum(BioNordika), penicillin-streptomycin (100 IU/ml) and $100 \mu \mathrm{g} / \mathrm{ml}$ respectively, Fisher Scientific), $10 \mathrm{mM}$ HEPES and $1 \mathrm{mM}$ Sodium Pyruvate (Fisher Scientific). In starvation medium, 
the concentration of Tet System approved fetal bovine serum was reduced from $10 \%$ to $0.5 \%$.Doxycycline (BioNordika) was added at concentrations indicated in the figures.

\section{Tet0n 3G Expression System in 22Rv1}

The TetOn3G expression system was used to over express YIPF6 in 22Rv1 cells as recommended by the vendor (Clontech Laboratories, Takara Bio). First, a parental 22Rv1 clone was constructed by transfecting 22Rv1 cells with the regulator plasmid pCMV-Tet3G. cDNA representing full length YIPF6 (variant A, 711 nucleotides),was amplified, using the primer pair 5'-GGC GGA TAT CAC GCG TAT GGC GGA AGC GGA GGA GTC TC-3' and 5'-CAG TTA CAT TGG ATC CTT AT GAG GAG TAA AGG TGA GAA TCA-3'. The PCR product was then cloned into the expression vector pTRE3GZsGreen 1 using In-Fusion PCR cloning technology as recommended by the vendor (Clontech). To get a clone with inducible expression of YIPF6, this expression plasmid was then transfected into the 22Rv1 parental cells containing the integrated regulator plasmid.

\section{Proliferation Assay}

$4 \times 10^{3}$ cells in $100 \mu \mathrm{l}$ were seeded per well in a 96 well plate and incubated overnight in ordinary medium to let the cells attach. Thereafter, the medium was exchanged to complete medium containing different concentrations of doxycycline (BioNordika) as specified in (Figure 1), and incubated for another $48 \mathrm{~h}$ before growth of the cells was analyzed using the Cell Proliferation Kit 1 (Roche, Germany) according to the manual. Absorbance was measured using an ELISA reader at $590 \mathrm{~nm}$, with reference wavelength 650 nm subtracted.
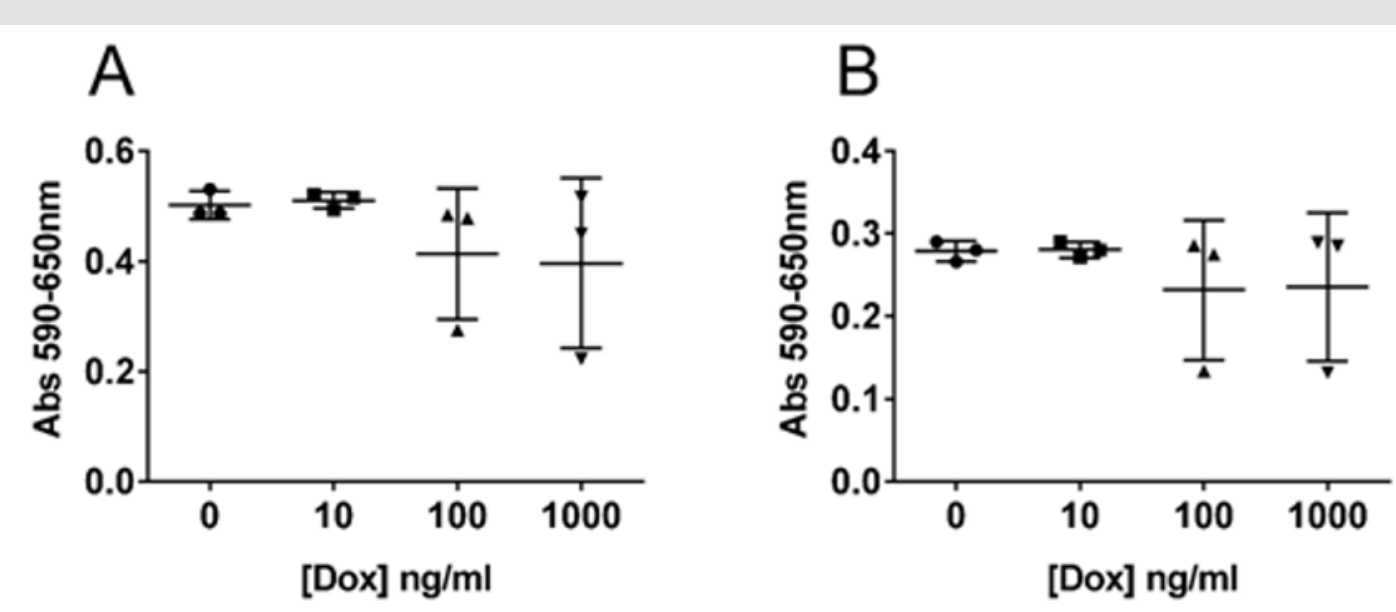

Figure 1: Influence of doxycycline on cell proliferation.

Note: 22Rv1 (A) and PC3 (B) cells were incubated with increasing concentrations of doxycycline (dox) as indicated in the figure, for $72 \mathrm{~h}$ before cell proliferation was measured using an ELISA reader. Absorbance was measured at 590nm, and reference wavelength was set to $650 \mathrm{~nm}$. The experiment was done in triplicate and the individual values are plotted in the figure as a scatter plot with mean and SD shown with bars.

\section{Migration and Invasion Assay}

For migration and invasion assays, 3.5x10522Rv1 cells, were seeded in $5 \mathrm{ml}$ volume into T25 flasks (Sarstedts) and preincubated for $24 \mathrm{~h}$ before adding fresh medium containing 0,10 or 100ng/ml doxycycline (BioNordika). After another $48 \mathrm{~h}$ the cells were starved overnight in starvation medium containing different concentrations of doxycycline as stated above. After a total of $72 \mathrm{~h}$ of induction the cells were trypsinated, washed in starvation medium and counted in a Countess automated cell counter (Invitrogen, ThermoFisher Scientific) before migration and invasion abilities of the cells were studied using BD BioCoat control inserts and Growth Factor Reduced MATRIGEL Invasion chambers (BD Biosciences) as recommended by the vendor. The cells were harvested after $24 \mathrm{~h}$.
Cells still located at the upper side of the chambers were washed away using a cotton tipped swab. The amount of migrating and invading cells were then quantified measuring absorbance at 550 $\mathrm{nm}$ after using staining and extraction solutions from Cell Biolabs migration assay according to manual (Cell BioLabsinc).

\section{Statistical Analysis}

Statistical significance was determined in multiple t-tests using the Holm Sidak method, with $\alpha=5.000 \%$ on the transfected $22 \mathrm{Rv} 1$ cells shown in (Figures 2A \& 2C). For the non-transfected 22Rv1 cells, shown in (Figures 2B \& 2D), we used an unpaired t-test. All analysis were performed using the GraphPad Prism version 6.07 for Windows, Graph Pad Software, La Jolla California USA, www. graphpad.com 

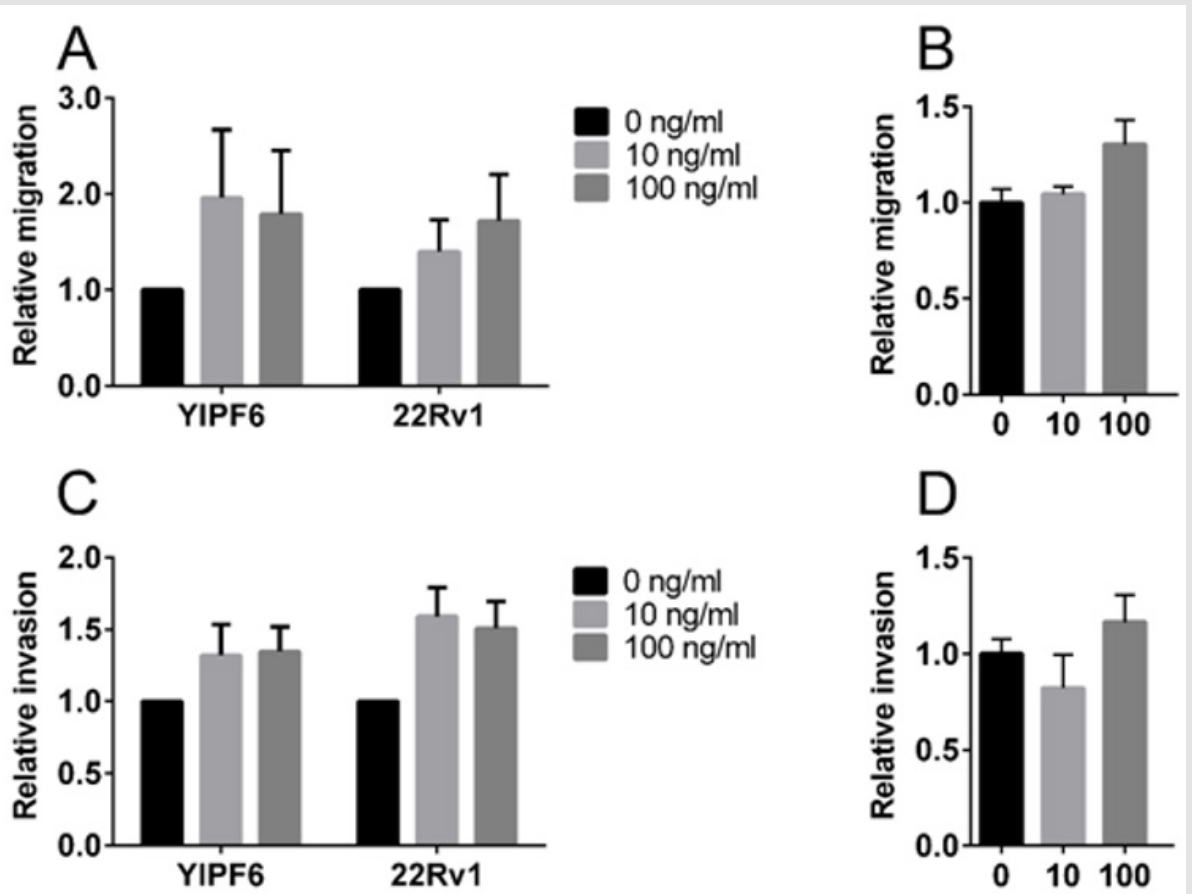

Figure 2: Effect of doxycycline on cell migration and invasion.

Note: Migration (A, B) and invasion (C, D) shown for 22Rv1 cells transfected with doxycycline-inducible plasmids (A, C) or non-transfected 22Rv1 cells (B, D). The insert in figure A and C, and the labels under the X-axes in B and D show the final doxycycline concentrations used in $\mathrm{ng} / \mathrm{ml}$. In each experiment, all results were related to the mean value obtained at $0 \mathrm{ng} / \mathrm{ml}$. The results are shown as mean with SD indicated as error bars.

\section{Results}

Doxycycline has an effect on cell proliferation in 22Rv1 and PC3 cell lines as can be seen in (Figure 1). No difference is observed between cells grown in the presence of $10 \mathrm{ng} / \mathrm{ml}$ doxycycline compared to the negative control. At $100 \mathrm{ng} / \mathrm{ml}$ and $1000 \mathrm{ng} / \mathrm{ml}$ you can see an increase in the variation of the proliferation rate which seems to increase with more dense cultures (data not shown). Doxycycline reduces the growth rate but do not affect the viability of the cells (Table 1). Induction of YIPF6 transfected 22Rv1 cells indicate that increased levels of YIPF6 increase the migration of the cells and to a lesser extent the invasive capacity, but including the results from the control parental 22Rv1 cells containing only the regulator plasmid indicate that this is an effect caused by doxycycline and not YIPF6 (Figure 2A \& 2C). (Figure 2B \& 2D) show the results from a follow up study where the effect of doxycycline was tested on non-transfected 22Rv1 cells. Analysis show statistical significant differences when comparing the invasion results for the transfected 22Rv1, with $\mathrm{p}=0,04$ comparing results for $10 \mathrm{ng} / \mathrm{ml}$ with negative control, and $\mathrm{p}=0,05$ comparing $100 \mathrm{ng} / \mathrm{ml}$ with the negative control. For the non-transfected $22 \mathrm{Rv} 1$ cells, significance was found for the migration when comparing $100 \mathrm{ng} / \mathrm{ml}$ with the negative control $(\mathrm{p}=0,02)$.
Table 1: Effect of doxycycline on growth rate.

\begin{tabular}{|c|c|c|}
\hline$[$ Dox] $\mathbf{n g} / \mathbf{m l}$ & No Viable Cells $/ \mathbf{m l}$ & Viability (\%) \\
\hline 0 & $2.4 \times 10^{6}$ & 97 \\
\hline 10 & $2.0 \times 10^{6}$ & 98 \\
\hline 100 & $1.6 \times 10^{6}$ & 98 \\
\hline
\end{tabular}

\section{Discussion}

Inducible expression systems are convenient to use when you want to study the effect of a certain gene product [1]. As soon as you have identified a clone that is stably expressing the DNA you have integrated, you can easily adjust the levels of expression by adding different concentrations of the inducible agent and study the effects in various assays. You have a perfect control in the cell line itself just by omitting the induction and thereby, you do not have to worry about that the phenotypic changes you recognize is due to differences in genetic background of the cells you are studying. In our lab, we used the Tet On 3 G expression system from Clontech in order to study the function of YIPF6, a gene that is co-amplified with the androgen receptor in a subset of bone metastasis from patients suffering from castration resistant prostate cancer [4]. Our experimental system was very sensitive, and we got a very nice induction using concentrations of doxycycline, as low as $10 \mathrm{ng} / \mathrm{ml}$, which is ten 
to hundred fold lower than the concentration recommended for screening for positive clones according to the manual (PT5148-1, Clontech). By using 10 to $100 \mathrm{ng} / \mathrm{ml}$ doxycycline we obtained levels of YIPF6 that very well corresponded to the levels of transcript found in patient tumor material (data not shown). As we wanted to mimic the physiologically relevant expression levels of YIPF6, this was the concentration of doxycycline we decided to use when we went on to do our migration and invasion assays. We obtained very interesting results showing that induced expression of YIPF6 had impact on the migratory ability of the cells (Figure 2A). A trend of increased invasion of the cells could also be seen (Figure 2C). But when we looked at the results from the 22Rv1 cells transfected with the regulatory plasmid, a control usually not included, we got quite concerned as those cells seemed to be as affected as the YIPF6 transfected clones showing statistically differences for the invasion already at $10 \mathrm{ng} / \mathrm{ml}$ of doxycycline $(\mathrm{p}=0,04)$. When we complemented these experiments using non-transfected 22Rv1 cells, we got a statistical significance with a $p=0,02$ when using 100 $\mathrm{ng} / \mathrm{ml}$ of doxycycline. Looking at the proliferation results in (Figure 1) at higher doxycycline concentrations it is obvious that the drug is affecting the cells in the range we were performing our experiments. There is also a trend that higher cell concentrations work in synergy with the effects of doxycycline as the variation of the experiments are more pronounced with higher cell concentrations in both 22Rv1 and PC3 cells (data not shown). Those observations altogether made us change our research strategy and use a non-inducible expression system in our further studies on YIPF6 [4]. In the study of Klokk et al they identified kallikrein-related peptidase 4 (KLK4), as a proliferative factor of prostate cancer cells that significantly altered cell cycle regulatory gene expression and they show a list of genes significantly altered with increasing KLK4 expression [5]. In these experiments, they used a doxycycline inducible virus system for the overexpression of KLK4. In our group, we have used two other systems to study the function of KLK4.

In PC3 cells, we have used pcDNA3.1 V5/His as the vector for over expressing KLK4. This is a vector expressing the inserted transcript under control of the constitutively active CMV promoter (Invitrogen). In 22Rv1 cells, we used the SMARTer Lentivirus shRNAsystem (Horizon Discoveries) to get a stable over expression of shKLK4 in order to reduce the levels of KLK4 transcript expressed (to be published elsewhere). When we compared our results to those of Klokk et al, we could not see any effect of the genes they listed as affected by KLK4 expression in our systems. When we looked at our control data from an Illumina expression array $[4,6]$, we could identify 3 genes (CCNH, CDKN2B and GADD45A) that were affected by just adding doxycycline to our $22 \mathrm{Rv} 1$ cells at 10 to 100 times lower concentrations than those used in the study of Klokk et al. This indicates that those results published could be a result of doxycycline treatment instead of changes in the KLK4 expression levels. It has been published before that human cell lines are affected by doxycycline [2,7], but in those studies higher concentrations of the tetracycline was used. In the work of Ahler et al [2] it is obvious that various cell lines differ in their sensitivity towards the drug, but interestingly, the most sensitive cell line they tested was LNCaP, also derived from human prostate cancer cells. Doxycycline and other tetracyclines have also been tested in cancer therapy [8].

\section{Conclusion}

Doxycycline induced expression systems are commonly used. Some cell lines are very sensitive to also very low concentrations of doxycycline, therefore it is of uttermost importance to include the correct controls before interpreting the results obtained.

\section{Acknowledgement}

Pernilla Wikström and Elin Thysell (Umeå University, Sweden) are acknowledged for generously sharing the Illumina expression data. Professor Wikström is also generously financing my position. Göran Brattsand has contributed with valuable comments on the manuscript. This study was supported by grants from Cancerforskningsfonden in Norrland and Lions Cancerforskningsfond at Umeå University.

\section{References}

1. Kallunki T, Barisic M, Jaattela M, Liu B (2019) How to Choose the Right Inducible Gene Expression System for Mammalian Studies? Cells 8(8): 796.

2. Ahler E, Sullivan WJ, Cass A, Braas D, York AG, et al. (2013) Doxycycline Alters Metabolism and Proliferation of Human Cell Lines. Plos One 8(5): e64561.

3. Van den Bogert C, van Kernebeek G, de Leij L, Kroon AM (1986) Inhibition of mitochondrial protein synthesis leads to proliferation arrest in the G1-phase of the cell cycle. Cancer Lett 32(1): 41-51.

4. Djusberg E, Jernberg E, Thysell E, Golovleva I, Lundberg P, et al. (2017) High levels of the AR-V7 Splice Variant and Co-Amplification of the Golgi Protein Coding YIPF6 in AR Amplified Prostate Cancer Bone Metastases. Prostate 77(6): 625-638.

5. Klokk TI, Kilander A, Xi Z, Waehre H, Risberg B, et al. (2007) Kallikrein 4 is a proliferative factor that is over expressed in prostate cancer. Cancer Res 67(11): 5221-5230.

6. Hornberg E, Ylitalo EB, Crnalic S, Antti H, Stattin P, et al. (2011) Expression of androgen receptor splice variants in prostate cancer bone metastases is associated with castration-resistance and short survival. PLoS ONE 6(4): e19059.

7. Fife RS, Sledge GW, Roth BJ, Proctor C (1998) Effects of doxycycline on human prostate cancer cells in vitro. Cancer Lett 127(1-2): 37-41.

8. Saikali Z, Singh G (2003) Doxycycline and other tetracyclines in the treatment of bone metastasis. Anticancer Drugs 14(10): 773-778. 
ISSN: 2574-1241

DOI: 10.26717/BJSTR.2020.28.004636

Maria Brattsand. Biomed J Sci \& Tech Res

(c) 9 This work is licensed under Creative

Submission Link: https://biomedres.us/submit-manuscript.php

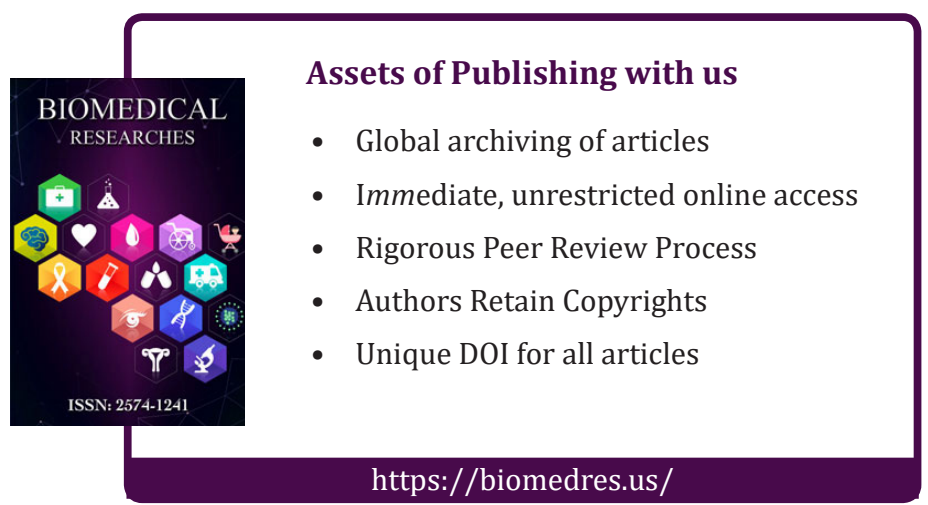

